\title{
Analytic pavement modelling with a fragmented layer
}

\section{Levenberg, Eyal; Skar, Asmus}

\section{Published in:}

The International Journal of Pavement Engineering

Link to article, DOI:

10.1080/10298436.2020.1790559

Publication date:

2020

Document Version

Early version, also known as pre-print

Link back to DTU Orbit

Citation (APA):

Levenberg, E., \& Skar, A. (2020). Analytic pavement modelling with a fragmented layer. The International Journal of Pavement Engineering. https://doi.org/10.1080/10298436.2020.1790559

\section{General rights}

Copyright and moral rights for the publications made accessible in the public portal are retained by the authors and/or other copyright owners and it is a condition of accessing publications that users recognise and abide by the legal requirements associated with these rights.

- Users may download and print one copy of any publication from the public portal for the purpose of private study or research.

- You may not further distribute the material or use it for any profit-making activity or commercial gain

- You may freely distribute the URL identifying the publication in the public portal

If you believe that this document breaches copyright please contact us providing details, and we will remove access to the work immediately and investigate your claim 


\title{
Analytic pavement modeling with a fragmented layer
}

Eyal Levenberg (corresponding author)

Department of Civil Engineering, Section for Geotechnics and Geology

Technical University of Denmark, Kgs. Lyngby, Denmark, 2800

Email: eylev@byg.dtu.dk

\author{
Asmus Skar \\ Department of Civil Engineering, Section for Geotechnics and Geology \\ Technical University of Denmark, Kgs. Lyngby, Denmark, 2800 \\ Email: asska@byg.dtu.dk
}




\title{
Analytic pavement modeling with a fragmented layer
}

\begin{abstract}
Layered elastic theory is a familiar and commonly accepted framework for modeling and analysis of pavement systems. In actuality however, the suitability of the theory is challenged whenever the system includes a fragmented layer - which violates the inherent layer continuity assumption. This paper addressed the modeling challenge in these situations by introducing a fragmented layer into the accepted framework. A new formulation was proposed, based on the hypothesis that the thickness of the fragmented layer can be incorporated as part of its governing parameters. Doing so allowed for treating the fragmented layer as a new kind of interface. Subsequently, the work focused on formulation development and clear step-by-step presentation of new derived expressions applicable to the case of a stratified half-space containing a fragmented layer. Several synthetic cases were generated and interrogated to verify the correctness of underlying equations and to demonstrate capabilities. Based on this interrogation the proposed formulation is deemed rich of prospective utility, especially for modeling pavements surfaced with concrete paving blocks, pavement systems that contain cracked-and-seated (or rubblized) Portland cement concrete, and aged or rehabilitated pavement systems that contain fatigued (multi-fractured) asphalt concrete layers.
\end{abstract}

Keywords: Pavement modeling; Layered elasticity; Fragmented layer; Small-element paving; cracked-and-seated Portland cement concrete; Fatigued asphalt concrete.

\section{Introduction}

Pavement systems are commonly modeled within a continuum mechanics framework by means of layered elastic theory (LET). This theory analytically resolves the mechanical responses in a stratified half-space due to vertical loading applied at the surface (Burmister 1943, Burmister 1945, Peutz et al. 1968). The typical modeling assumptions include homogenous and weightless layers, as well as isotropic linear-elastic material behavior. Such modeling approach is widespread, first and foremost because of the ability to capture and reproduce key response features with a relatively small set of input parameters (Levenberg et al. 2009). Additionally, LET is often utilized in inverse analysis schemes because of its excellent computational efficiency (Khazanovich and Wang 2007, Andersen et al. 2018). Another reason for favoring LET is the relative ease at which it can be extended to 
accommodate more realistic circumstances, e.g., viscoelastic behavior (Levenberg 2016), material anisotropy (Singh 1986, Pan 1989, Levenberg 2009), horizontal loadings (Kai 1987, Maina and Matsui 2004), and imposed subterranean displacements (Levenberg 2013, Skar et al. 2018).

Due to the material continuity assumption, LET is fundamentally applicable to the modeling of pavement layers that are either in pristine condition or pavement layers that are aged but otherwise unbroken. However, actual pavement systems very often contain at least one fragmented layer (FL) - challenging the suitability of LET. Perhaps the most common example for this situation in new constructions is small-element paving technologies, wherein the top pavement layer is essentially discontinuous, composed of individual interlocking (precast) concrete blocks (Jamshidia et al. 2019). Another common example for this situation is fatigued in-service asphalt pavements containing a densely cracked asphalt concrete (AC) layer. For rehabilitated constructions, pavement systems containing a FL arise whenever new AC is directly paved over an existing (aged) AC layer that is severely distressed. In this case, the new continuous layer of AC is supported from below by a multifractured layer that may be deteriorated to the point of being a mere collection of abutting material blocks. Yet another common rehabilitation example refers to the case of paving AC over an existing cracked-and-seated Portland cement concrete (PCC) pavement. This so-called fractured-slab technology involves purposeful breaking of an existing PCC into small pieces to deter reflection cracks in the overlay (Witczak and Rada 1992, Bemanian and Sebaaly 1999, Freeman 2002).

As a means of modeling the above-mentioned situations, two approaches are currently being taken by the engineering community. The first is utilizing LET, but with reduced elastic properties for the FL (Ahrlich 1989, Kasahara and Matsuno 1988). Doing so involves the difficulty of identifying equivalent elasticity constants for representing a multi-fractured layer, but preserves the familiar analysis framework with all above listed advantages. At the same time, calculation results are biased; this is because a densely cracked layer, unlike a continuous layer, can offer considerable vertical rigidity yet negligibly small rigidity in bending mode. Moreover, a discontinuous layer can exhibit some substantial rigidity in transverse shear mode given that the individual material blocks are often touching along their sides.

The second approach for dealing with a FL abandons the analytic theory in favor of purely numerical engines such as the Finite Element (FE) method. Within a FE framework, one modeling method is treating the FL as a collection of material blocks, each with a given geometry, as well as some presumed interaction mechanism between the blocks (Nishizawa 2003, Molenaar et al. 1984, Skar and Poulsen 2015). Another modeling method involves the theory of continuum damage 
mechanics, which deals with the behavior of a progressively deteriorating medium due to the growth of many randomly distributed micro cracks (Kachanov 1986, Schapery 1999). Continuum damage mechanics has been recently applied to model top-down and fatigue cracking in asphalt concrete pavements (Baek et al. 2012, Kutay and Lanotte 2018).

While advanced numerical schemes are fundamentally superior to LET, they do not enjoy widespread adaption among practitioners, and are yet to be accepted universally among researchers. This is mainly due to the high computational cost involved, as well as the large number of material functions and input parameters required for the simulation. Therefore, the work herein pursues the introduction of a FL into the familiar LET-based framework. The focus is on formulation development and step-by-step presentation of newly derived expressions, so that they can be reproduced and coded by others. The paper commences by restating the governing equations of LET (see Subsection 2.1 and Appendix A); this is followed by a description of the proposed modeling development (see Subsection 2.2 and Appendix B). Then, in Section 3, example cases are generated to demonstrate capabilities and highlight differences compared to the standard LET. Finally, in Section 4, prospective utility cases are pointed out and discussed.

\section{Modeling}

\subsection{Standard formulation}

The standard LET formulation is axisymmetric; it involves a semi-infinite medium composed of $I-1$ parallel (horizontal) layers overlying a half-space - see Figure 1a. The layers are numbered serially, with the top layer being Layer 1 and the half-space, Layer $I$. Each layer is characterized by a Young's modulus $E_{i}$, Poisson's ratio $v_{i}$, and thickness $h_{i}$, with the subscript $i$ serving as a layer identifier. A cylindrical coordinate system is introduced such that its origin is positioned at the surface of Layer 1 ; the vertical $z$-axis is drawn into the medium, and the radial $r$-axis is parallel to the top boundary. The depth from the surface to the interface separating layers $i$ and $i+1$ is denoted by $z_{i}$. Accordingly, $z_{1}$ is the thickness of Layer 1 (i.e., $z_{1}=h_{1}$ ), $z_{2}$ is the combined thickness of Layers 1 and 2 (i.e., $\left.z_{2}=h_{1}+h_{2}\right)$, and so on. The collective thickness of all $I-1$ layers is denoted by $H$, i.e., $H=z_{I-1}=h_{1}+h_{2}+\ldots+h_{I-1}$.

Vertical loading representing tire-pavement contact interaction is applied at the surface (i.e., top of Layer 1), just above the coordinate system origin. It entails a uniform stress with intensity $q_{0}$ operating over a circular area with radius $a$. Such loading is mathematically expressed as: 
$q(r)=0.5 q_{0}+0.5 q_{0} \operatorname{sgn}(a-r)$ where $\operatorname{sgn}(\cdot)$ is the signum function. Based on the Hankel transform theory, a mathematically equivalent expression for $q(r)$ is

$$
q(r)=q_{0} \bar{a} \int_{m=0}^{\infty} J_{0}(m \bar{r}) J_{1}(m \bar{a}) d m
$$

where $\bar{a}=a / H$ is a dimensionless loading radius, $\bar{r}=r / H$ is a dimensionless radial coordinate, $J_{0}(\cdot)$ and $J_{1}(\cdot)$ are Bessel functions of the first kind of order zero and one (respectively), and $m$ is a unitless positive integration parameter (Hankel parameter or wave number).

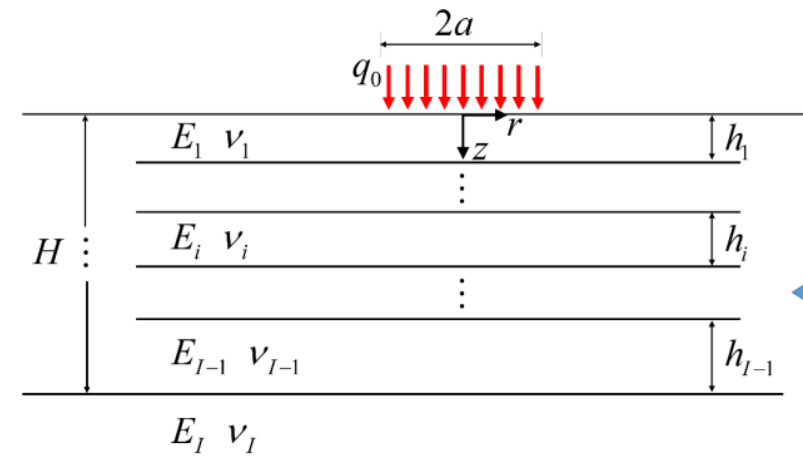

(a)

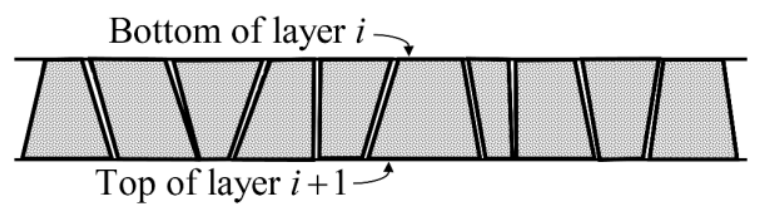

(b)

Figure 1. Cross sectional view of: (a) a standard layered elastic pavement model, and (b) an illustration of a FL inserted between continuous layers $i$ and $i+1$.

The medium displacements in the $z$ and $r$ directions for layer $i$ are denoted by $\left(u_{z}\right)_{i}$ and $\left(u_{r}\right)_{i}$ respectively; due to axisymmetry there are no displacements in the $\theta$ direction. The relevant stress-strain (constitutive) equations are

$$
\begin{aligned}
& \left(\varepsilon_{z}\right)_{i}=\frac{1}{E_{i}}\left(\sigma_{z}\right)_{i}-\frac{v_{i}}{E_{i}}\left(\sigma_{\theta}\right)_{i}-\frac{v_{i}}{E_{i}}\left(\sigma_{r}\right)_{i} \\
& \left(\varepsilon_{r}\right)_{i}=\frac{1}{E_{i}}\left(\sigma_{r}\right)_{i}-\frac{v_{i}}{E_{i}}\left(\sigma_{\theta}\right)_{i}-\frac{v_{i}}{E_{i}}\left(\sigma_{z}\right)_{i} \\
& \left(\varepsilon_{\theta}\right)_{i}=\frac{1}{E_{i}}\left(\sigma_{\theta}\right)_{i}-\frac{v_{i}}{E_{i}}\left(\sigma_{r}\right)_{i}-\frac{v_{i}}{E_{i}}\left(\sigma_{z}\right)_{i} \\
& \left(\varepsilon_{r z}\right)_{i}=\frac{1+v_{i}}{E_{i}}\left(\tau_{r z}\right)_{i}
\end{aligned}
$$

where $\left(\varepsilon_{z}\right)_{i},\left(\varepsilon_{r}\right)_{i},\left(\varepsilon_{\theta}\right)_{i}$ and $\left(\varepsilon_{r z}\right)_{i}$ are the strains in $z, r, \theta$, and $r-z$ directions for layer $i$, and $\left(\sigma_{z}\right)_{i},\left(\sigma_{r}\right)_{i},\left(\sigma_{\theta}\right)_{i}$, and $\left(\tau_{r z}\right)_{i}$ are the respective stresses. 
Based on the work of Love (1927) (see also Burmister 1945), the following expressions provide the stresses and displacements

$$
\begin{aligned}
& \left(\sigma_{z}\right)_{i}=\frac{\partial}{\partial z}\left(\left(2-v_{i}\right) \nabla^{2} \varphi_{i}-\frac{\partial^{2} \varphi_{i}}{\partial z^{2}}\right) \\
& \left(\sigma_{r}\right)_{i}=\frac{\partial}{\partial z}\left(v_{i} \nabla^{2} \varphi_{i}-\frac{\partial^{2} \varphi_{i}}{\partial r^{2}}\right) \\
& \left(\sigma_{\theta}\right)_{i}=\frac{\partial}{\partial z}\left(v_{i} \nabla^{2} \varphi_{i}-\frac{1}{r} \frac{\partial \varphi_{i}}{\partial r}\right) \\
& \left(\tau_{r z}\right)_{i}=\frac{\partial}{\partial r}\left(\left(1-v_{i}\right) \nabla^{2} \varphi_{i}-\frac{\partial^{2} \varphi_{i}}{\partial z^{2}}\right) \\
& \left(u_{z}\right)_{i}=-\frac{1+v_{i}}{E_{i}}\left(\left(1-2 v_{i}\right) \nabla^{2} \varphi_{i}+\frac{\partial^{2} \varphi_{i}}{\partial r^{2}}+\frac{1}{r} \frac{\partial \varphi_{i}}{\partial r}\right) \\
& \left(u_{r}\right)_{i}=\frac{1+v_{i}}{E_{i}}\left(\frac{\partial^{2} \varphi_{i}}{\partial r \partial z}\right)
\end{aligned}
$$

where $\varphi_{i}=\varphi_{i}(r, z)$ is a scalar potential function (units of forcexlength), and $\nabla^{2}=\partial^{2} / \partial r^{2}+r^{-1} \partial / \partial r+\partial^{2} / \partial z^{2}$ is the axisymmetric Laplace operator in $r$ and $z$. A suitable expression for such a potential function is

$$
\varphi_{i}=q_{0} \bar{a} H^{3} \int_{m=0}^{\infty} \frac{J_{0}(m \bar{r}) J_{1}(m \bar{a})}{m^{3}}\left(\begin{array}{l}
A_{i} e^{-m\left(\bar{z}_{i}-\bar{z}\right)}-B_{i} e^{-m\left(\bar{z}-\bar{z}_{i-1}\right)} \\
+C_{i} m \bar{z} e^{-m\left(\bar{z}_{i}-\bar{z}\right)}-D_{i} m \bar{z} e^{-m\left(\bar{z}-\bar{z}_{i-1}\right)}
\end{array}\right) d m
$$

in which $\bar{z}=z / H$ and $\bar{z}_{i}=z_{i} / H$ are dimensionless entities (with $\bar{z}_{0}=0$ and $\bar{z}_{I}=\infty$ ), while $A_{i}=A_{i}(m), B_{i}=B_{i}(m), C_{i}=C_{i}(m)$, and $D_{i}=D_{i}(m)$ are unitless functions that embody the layered system connectivity and properties. Upon insertion of $\varphi_{i}$ into Equations (6)-(11), explicit expressions are obtained for calculating stresses and displacements anywhere within the layered medium; these are outlined in Appendix A.

The proposed $\varphi_{i}$, along with all derived equations that follow, are essentially the same as in Burmister (1945) although they may appear slightly different. Specifically, $\varphi_{i}$ is a combination between the potential function in Van Cauwelaert (2003) and the idea advocated in Huang (2004) of introducing non-dimensional depth coordinate $\bar{z}$, radial coordinate $\bar{r}$, and radius of loaded area $\bar{a}$. 
The main advantage of working with normalized parameters is numerical stability and consistency of results across different unit systems.

For $i=1,2, \ldots, I$ the system connectivity functions $A_{i}, B_{i}, C_{i}$, and $D_{i}$ are obtained by imposing boundary conditions. First, for the surface of the top layer, where $i=1$ and $\bar{z}=0$, vertical stress must equal the applied circular stress distribution, and shear stresses must vanish everywhere

$$
\left(\sigma_{z}\right)_{1}=q(r) \text { and }\left(\tau_{r z}\right)_{1}=0
$$

Second, at the bottom of layer $i$ and at the top of layer $i+1$, i.e., at the interface where $\bar{z}=\bar{z}_{i}$, vertical stresses, vertical displacements, shear stresses, and radial displacements are identical. Formally, this means $\left(\sigma_{z}\right)_{i}=\left(\sigma_{z}\right)_{i+1},\left(u_{z}\right)_{i}=\left(u_{z}\right)_{i+1},\left(\tau_{r z}\right)_{i}=\left(\tau_{r z}\right)_{i+1}$, and $\left(u_{r}\right)_{i}=\left(u_{r}\right)_{i+1}$. The resulting equation set takes the form

$$
\mathbf{M} \cdot\left(\begin{array}{llll}
A_{i} & B_{i} & C_{i} & D_{i}
\end{array}\right)^{\mathrm{T}}+\mathbf{N} \cdot\left(\begin{array}{llll}
A_{i+1} & B_{i+1} & C_{i+1} & D_{i+1}
\end{array}\right)^{\mathrm{T}}=\mathbf{0}
$$

where $\mathbf{M}$ and $\mathbf{N}$ are $4 \times 4$ matrices, outlined in Appendix B. Third, the sought LET solution must embody the requirement that all responses vanish for $i=I$ as the evaluation depth approaches infinity, i.e., $\bar{z} \rightarrow \infty$. This condition results in

$$
A_{I}(m)=0 \text { and } C_{I}(m)=0
$$

Thus, solving Equations (13)-(15) provides the connectivity functions for any $m$ value. Then after, by integration with respect to $m$, stresses and displacements are determined from Equations (A.1)(A.6), see Appendix A. Finally, strains are calculated from Equations (2)-(5).

\subsection{Fragmented layer formulation}

Consider a FL comprised of, e.g., concrete paving blocks, cracked AC, or cracked-and-seated PCC. A generic cross-section of such a layer is illustrated in Figure $1 \mathrm{~b}$ as a collection of abutting material blocks of different sizes. This FL is inserted and positioned in-between layers $i$ and $i+1$ of a stratified half-space complying with the usual LET assumptions. As can be seen, the FL bottom is in contact with the top of layer $i+1$, while the FL top is in contact with the bottom of layer $i$. Thus, according to this arrangement, simulating a FL that resides at the very top of a pavement system can be approached by placing it second from the top, below a layer of negligibly small thickness.

It should be understood that the individual fragments shown in Figure $1 \mathrm{~b}$ are merely a graphical illustration. In effect, the treatment being offered considers the FL as having smeared 
mechanical characteristics - thus avoiding the need to define material block dimensions and interconnectivity conditions. In general terms, the validity of such treatment is closely related to applying the representative volume element concept (Hill 1963), which under certain conditions permits the modeling of a heterogeneous material (or body) as homogenous. Accordingly, in order for the smeared properties to be representative, a FL should contain sufficiently small elements, especially with respect to the applied boundary conditions (e.g., diameter of the loaded zone in case the FL is close to the surface). While an exhaustive investigation and quantification of this aspect diverges from this paper's objective and scope, some limited size-effect analysis is included as part of the example cases in Section 3.

Hereafter, two possible mechanical analogs are suggested for representing a FL. The first, shown in Figure 2a, is referred to as P-type layer; it utilizes a Winkler-type spring-bed characterized by $k$ (units of force/length ${ }^{3}$ ) to represent vertical stiffness offered by the material blocks. The interaction between the individual blocks is introduced via two identical Pasternak-like shear layers, one connecting the top ends of the springs and the other connecting the bottom ends of the springs. These shear layers are treated as vertically incompressible, each providing resistance to shear deformation characterized by $0.5 G$ (units of force/length). The second type of FL representation is referred to as K-type. As shown in Figure 2b, it comprises of two identical Winkler-type spring-beds, each characterized by a constant $2 k$ (units of force/length ${ }^{3}$ ). Both the upper and lower spring-beds are connected to a Pasternak-like shear layer characterized by a shear deformation resistance $G$ (units of force/length). In addition to the above described components, and as can be seen in Figure 2, both the P-type and K-type representations include a set of horizontally oriented springs - characterized by $k_{h}$ (units of force/length ${ }^{3}$ ). These springs embody the ability of the FL to transfer parallel-oriented shear stresses that are operating at the top or bottom interfaces.

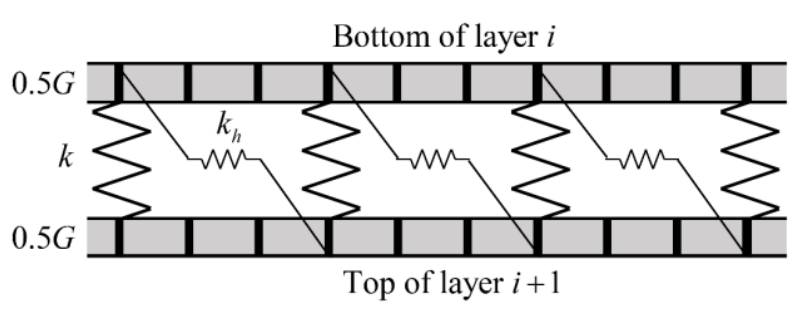

(a)

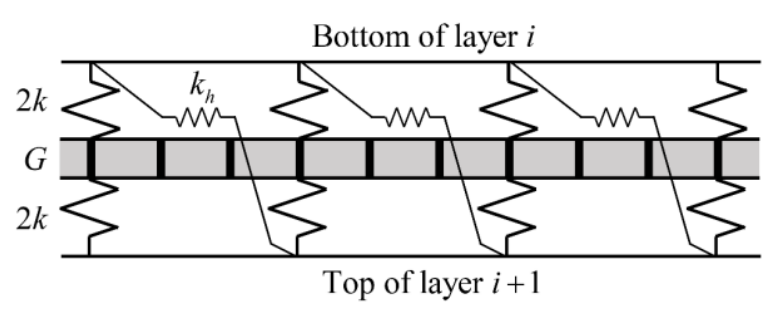

(b)

Figure 2. Two modeling options for a FL: (a) P-type representation, and (b) K-type representation.

The two proposed FL representations do not include layer thickness as a characteristic parameter. Such parameter is redundant from a modeling standpoint because the thickness can be 
indirectly incorporated in the values of the governing parameters $k, G$, and $k_{h}$ (Ioannides and Khazanovich 1998). For example, the value of $k$ can be estimated as the elastic modulus of the individual material blocks comprising the FL divided by the FL thickness. The values of $G$ and $k_{h}$ may be related to some average geometry of the individual fragments and to the gaps between them. Thus, the introduction of a FL into a pavement model based on LET can be done by reformulating the interface conditions between layer $i$ and layer $i+1$ (where the FL is introduced). Accordingly, the FL herein is not treated as an additional layer but rather as a new interface type. This approach is also justified given that there is no meaning to stresses and strains within the FL itself.

Consequently, the required changes to the standard LET formulation will only affect the elements of the $\mathbf{M}$ and $\mathbf{N}$ matrices in Equation (14). Considering first the P-type representation shown in Figure 2a, the following equation set is proposed for the corresponding interface between layer $i$ and layer $i+1$ where $\bar{z}=\bar{z}_{i}$

$$
\begin{aligned}
& \left(\sigma_{z}\right)_{i}-k\left(\left(u_{z}\right)_{i}-\left(u_{z}\right)_{i+1}\right)+0.5 G \nabla_{r}^{2}\left(u_{z}\right)_{i}=0 \\
& \left(\sigma_{z}\right)_{i+1}-k\left(\left(u_{z}\right)_{i}-\left(u_{z}\right)_{i+1}\right)-0.5 G \nabla_{r}^{2}\left(u_{z}\right)_{i+1}=0 \\
& \left(\tau_{r z}\right)_{i}=\left(\tau_{r z}\right)_{i+1} \\
& \left(\tau_{r z}\right)_{i}-k_{h}\left(\left(u_{r}\right)_{i}-\left(u_{r}\right)_{i+1}\right)=0
\end{aligned}
$$

Equation (16) represents vertical stress equilibrium at the top of the FL and bottom of layer $i$, while Equation (17) represents vertical stress equilibrium at the bottom of the FL and top of layer $i+1$; in both equations $\nabla_{r}^{2}=\partial^{2} / \partial r^{2}+r^{-1} \partial / \partial r$ (i.e., axisymmetric Laplace operator in $r$ ). Equation (18) embodies the assumption that shear stresses are equally distributed (spatially) across the top and bottom of the FL. The legitimacy of this assumption is based on a separate analysis in which the FL was modeled as a transversely-isotropic layer with a high vertical to horizontal modulus ratio. Regardless, the precision of this assumption improves with decreasing FL thickness. Lastly, Equation (19) represents the contribution of the horizontal springs; it relates the shear stress magnitude to the difference in radial displacement between the top and bottom of the FL (Goodman and Popov 1968, De Jong et al. 1973). Given in Appendix B is a matrix form of Equations (16)-(19), representing a Ptype FL model (to be used in place of $\mathbf{M}$ and $\mathbf{N}$ in Equation (14)).

As for the K-type interface shown in Figure 2b, the first two equations in the above set, i.e., Equations (16) and (17), should be replaced with 


$$
\begin{aligned}
& \left(\sigma_{z}\right)_{i}-0.25(G / k) \nabla_{r}^{2}\left(\sigma_{z}\right)_{i}-k\left(\left(u_{z}\right)_{i}-\left(u_{z}\right)_{i+1}\right)+0.5 G \nabla_{r}^{2}\left(u_{z}\right)_{i}=0 \\
& \left(\sigma_{z}\right)_{i+1}-0.25(G / k) \nabla_{r}^{2}\left(\sigma_{z}\right)_{i+1}-k\left(\left(u_{z}\right)_{i}-\left(u_{z}\right)_{i+1}\right)-0.5 G \nabla_{r}^{2}\left(u_{z}\right)_{i+1}=0
\end{aligned}
$$

Equation (20) denotes vertical stress equilibrium at the top of the K-type FL and bottom of (continuous) layer $i$; Equation (21) represents vertical stress equilibrium at the bottom of the K-type FL and top of (continuous) layer $i+1$. Given in Appendix B is a matrix form of Equations (20), (21), (18), and (19), representing a K-type FL model (to be used in place of $\mathbf{M}$ and $\mathbf{N}$ in Equation (14)).

In both the P-type and K-type FL formulations, the extreme case of $k_{h}=0$ means $\left(\tau_{r z}\right)_{i}=\left(\tau_{r z}\right)_{i+1}=0$, while the limit case of $k_{h} \rightarrow \infty$ means $\left(u_{r}\right)_{i}=\left(u_{r}\right)_{i+1}$. Moreover, setting $G=0$ forces the condition that $\left(\sigma_{z}\right)_{i}=\left(\sigma_{z}\right)_{i+1}$; this can be seen in the P-type formulation when subtracting Equation (17) from Equation (16), or in the K-type formulation when subtracting Equation (21) from Equation (20). Therefore, if $G=0$ is combined with $k_{h}=0$, then both FL representations reduce to a pure Winkler-type layer with spring-bed rigidity $k$ (and inability to transfer shear). Such setting may be most suited for representing a situation where the material blocks within the FL are thin, tall, and completely separated from each another. Lastly, the limit case of $k \rightarrow \infty$ essentially forces the condition that $\left(u_{z}\right)_{i}=\left(u_{z}\right)_{i+1}$; after division by $k$, this can be seen for the P-Type formulation in either Equation (16) or Equation (17), and for the K-type formulation in either Equation (20) or Equation (21). Therefore, setting $k \rightarrow \infty$ and $k_{h} \rightarrow \infty$ combined with $G=0$ effectively removes the FL from the system and produces a fully bonded (standard) LET interface between layer $i$ and layer $i+1$.

\section{Demonstration and verification}

\subsection{Parametric interrogation}

The new theory is interrogated hereafter for the purpose of demonstrating capabilities, and also verifying that the formulation (and coding) are error free. As a starting point, the two pavement systems shown in Figure 3 are parametrically interrogated; both systems are loaded over a single circular area with radius $a=150 \mathrm{~mm}$ and stress intensity $q_{0}=0.5 \mathrm{MPa}$. Figure $3 \mathrm{a}$ shows a model consisting of (top to bottom): a $50 \mathrm{~mm}$ thick layer with Young's modulus of $3000 \mathrm{MPa}$ overlaying a FL (having undefined thickness) that is supported on a $400 \mathrm{~mm}$ thick layer with Young's modulus of $200 \mathrm{MPa}$. The bottom half-space is characterized by a Young's modulus of $80 \mathrm{MPa}$. This system can represent a cracked-and-seated (or rubblized) PCC or a deteriorated (multi-fractured) asphalt pavement that received a $50 \mathrm{~mm}$ AC overlay. Figure $3 \mathrm{~b}$ is a variant of the aforementioned system in that the thickness of the top layer is reduced to $0.1 \mathrm{~mm}$. This ultrathin top layer means that the FL, 
which is placed second from the top, is effectively at the very top. Such a system can represent, e.g., an interlocking concrete block pavement.

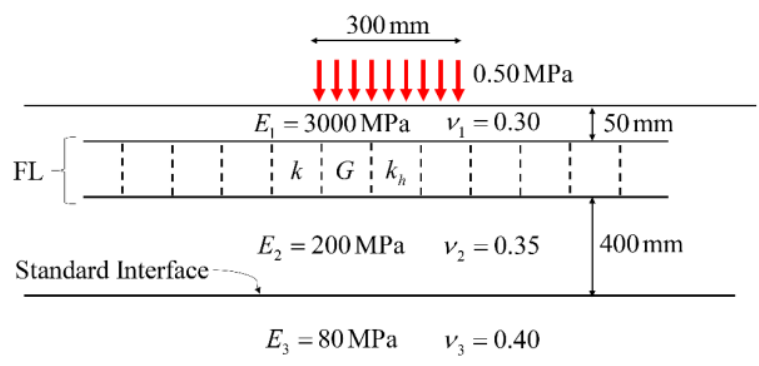

(a)

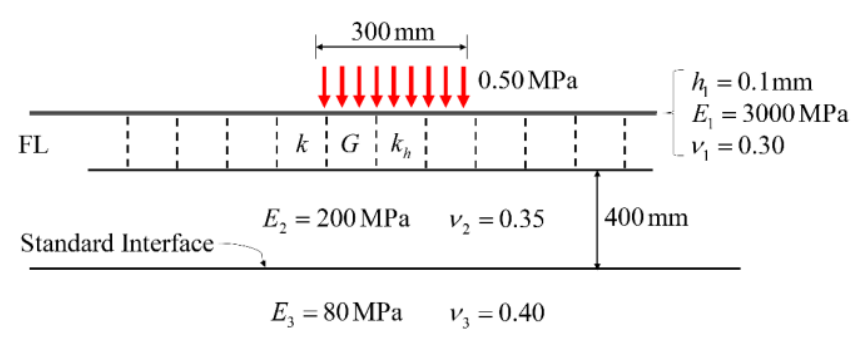

(b)

Figure 3. Cross-sectional view of two pavement systems for parametric model interrogation: (a) FL covered by a continuous layer, and (b) FL effectively at the very top.

The demonstration is focused on the vertical surface displacement profile, i.e., $\left(u_{z}\right)_{1}$ at $z=0$ ; this is done for radial offsets in the range of $r=0$ to $r=600 \mathrm{~mm}$, i.e., $0 \leq r \leq 4 a$. Capabilities are mapped by presenting results across a wide range of $k$ and $G$ values, and for two extreme $k_{h}$ values, namely $k_{h}=0$ and $k_{h} \rightarrow \infty$. Calculations involved analytical solution of Equations (13)-(15) in order to obtain the system connectivity functions $A_{1}(m), B_{1}(m), C_{1}(m)$, and $D_{1}(m)$; this was followed with numerically integrating Equation (A.5) after setting $i=1$ and $z=0$. This latter step was accomplished by partitioning the semi-infinite integration range into a finite sequence of 500 definite integrals, each spanning two consecutive integrand roots (Longman 1956). The definite integrals were evaluated with a 30-point Gauss quadrature formula.

Figure 4 addresses the pavement model of Figure 3a; it depicts calculated surface displacements for both a P-Type FL (Figures 4a and 4b) and K-Type FL (Figures 4c and 4d). Dashed lines represent calculations with $k_{h}=0$ and solid lines represent $k_{h} \rightarrow \infty$. The sensitivity to different $k$ values in the range of $k=0.1 \mathrm{MPa} / \mathrm{mm}$ up to $k \rightarrow \infty$ is displayed for $G=0$ (Figures $4 \mathrm{a}$ and $4 \mathrm{c}$ ), and separately for $G=10^{4} \mathrm{~N} / \mathrm{mm}$ (Figures $4 \mathrm{~b}$ and $4 \mathrm{~d}$ ). As can be seen, and as expected, displacement magnitudes increase for a given $G$ with decreasing $k$; they are slightly smaller for the $k_{h}=0$ case compared to the $k_{h} \rightarrow \infty$ case. Also, Figures $4 \mathrm{a}$ and $4 \mathrm{c}$ are identical, verifying that both the P-Type and K-Type representations yield identical results when $G=0$. It was moreover verified (separately) that the case of $G=0, k \rightarrow \infty$, and $k_{h} \rightarrow \infty$ essentially reproduces the displacements in a standard LET model where the FL does not exist, and the interface between Layer 1 and Layer 2 is perfectly bonded. By contrasting Figure $4 \mathrm{~b}$ and Figure $4 \mathrm{~d}$ it can be observed that for the same choice of $G$ the displacement magnitudes are more sensitive to $k$ values in the K-Type FL. A final verification test 
for the pavement system in Figure 3a involved considerably increasing the Young's modulus of Layer 2 and Layer 3 (i.e., $E_{2} \rightarrow \infty$ and $E_{3} \rightarrow \infty$ ), while at the same time setting $G=0$ and $k_{h}=0$. This case reduced the problem to an elastic layer resting on a Winkler foundation for which solutions are known (Kerr 1964, Dempsey et al. 1991).
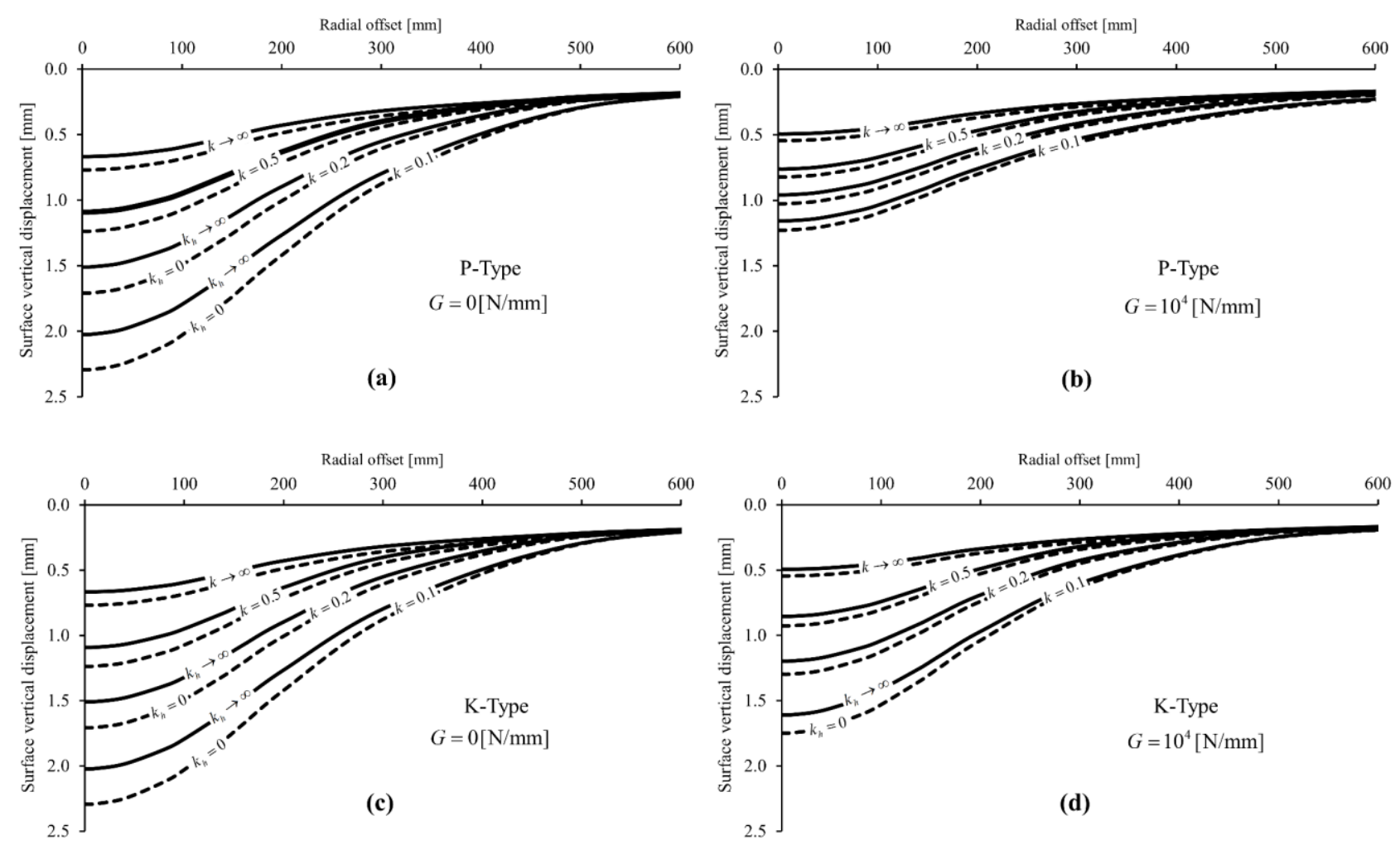

Figure 4. Calculated vertical surface displacements for the pavement system in Figure 3a with either P-Type FL ( $\mathrm{a}$ and b) and with a K-type FL (c and d). Two shear deformation resistances $(G)$ and four different values of the spring-bed constant $(k)$ are considered; units of $k$ are $\mathrm{MPa} / \mathrm{mm}$. Solid lines represent $k_{h} \rightarrow \infty$ and dashed lines represent $k_{h}=0$.

Figure 5 provides results for the pavement model in Figure 3b, where the FL is essentially at the top. It depicts calculated surface displacements for both a P-Type FL (Figures 5a and 5b) and KType FL (Figures 5c and 5d). The sensitivity to different $k$ values in the range of $k=0.1 \mathrm{MPa} / \mathrm{mm}$ up to $k \rightarrow \infty$ is displayed for $G=0$ (Figures $5 \mathrm{a}$ and $5 \mathrm{c}$ ) and separately for $G=10^{3} \mathrm{~N} / \mathrm{mm}$ (Figures $5 \mathrm{~b}$ and $5 \mathrm{~d}$ ). The case of $G=0$ is expected to reduce both FL types into a Winkler layer characterized by spring-bed constant $k$. This outcome is verified by observing that Figures $5 \mathrm{a}$ and $5 \mathrm{c}$ are identical. As expected in this case, the surface displacements exhibit discontinuity at the edge of the loaded area (radial offset of $150 \mathrm{~mm}$ ) with overall magnitudes that decrease with increasing $k$ values. Also, the case of $k \rightarrow \infty$ and $G=0$ was verified to reproduce the displacements in a standard LET model where the FL does not exist, and the loading is applied directly onto the layer below. 

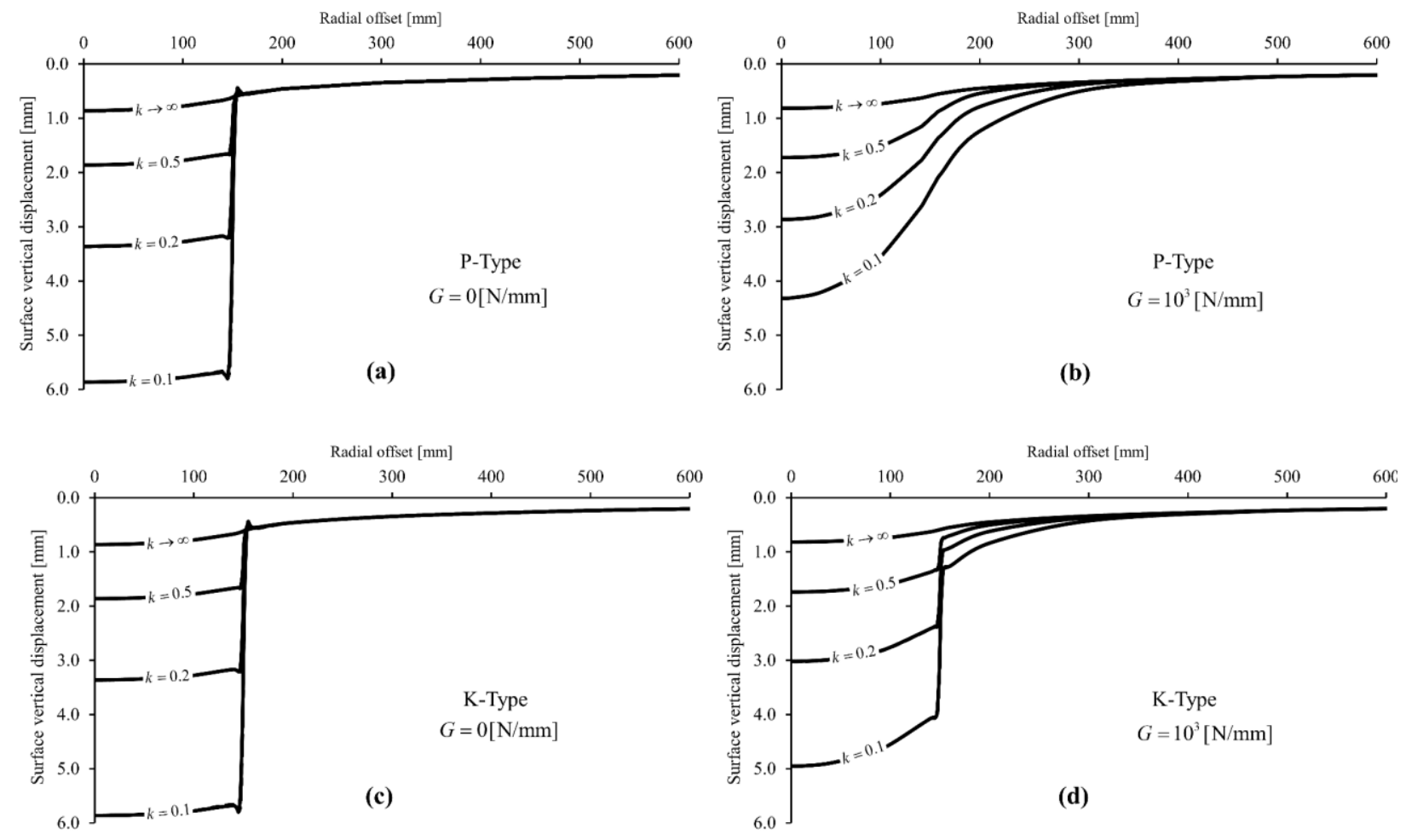

Figure 5. Calculated vertical surface displacements for the pavement system in Figure $3 \mathrm{~b}$ with either P-Type FL (a and b) and with a K-type FL (c and d). Two shear deformation resistances $(G)$ and four different values of the spring-bed constant $(k)$ are considered; units of $k$ are $\mathrm{MPa} / \mathrm{mm}$. Lines represent $k_{h} \rightarrow \infty$ and also $k_{h}=0$.

Additionally, by contrasting Figure $5 b$ with Figure $5 d$ it can be seen that the P-type and Ktype FLs behave differently when the shear interaction characteristic $G$ is greater than zero. In the P-type case, continuous behavior of the surface displacement with respect to the radial offset is imposed for $G>0$, while discontinuous behavior is permitted (and simulated) in the K-type case. These observations are perfectly in tune with the expected behavior considering the assumptions in Figure 2. Yet another verification test involved considerably increasing the Young's modulus of Layer 2 and Layer 3 (i.e., $E_{2} \rightarrow \infty$ and $E_{3} \rightarrow \infty$ ), and observing that the surface displacement shape matches the corresponding foundation model response (Kerr 1964). For the case of a P-type FL, a match was attained with a Pasternak foundation model characterized by a spring-bed coefficient $k$ and shear interaction $0.5 G$. For the case of a K-type FL, a match was attained with a Kerr foundation model characterized by shear interaction $G$ and identical upper and lower spring-bed coefficients $2 k$ . Lastly, it is noted that no dashed lines appear in Figure 5; this is because the case $k_{h}=0$ gives the exact same results as $k_{h} \rightarrow \infty$. Such outcome is expected whenever a FL is placed at the very top and loaded vertically. 


\subsection{Comparison with a numerical model}

Another demonstration of the potential utility of the new formulation deals with the ability to model a pavement system surfaced with concrete paving blocks. For this purpose, a reference linear elastic FE model was prepared in ABAQUS. This model consisted of a square block-paved area of dimensions $2630 \mathrm{~mm} \times 2630 \mathrm{~mm}$ composed of $961(=31 \times 31)$ identical blocks. Each block was 80 $\mathrm{mm} \times 80 \mathrm{~mm} \times 80 \mathrm{~mm}$ in size, and characterized by a Young's modulus of $30 \mathrm{GPa}$ and Poisson's ratio of 0.20 . The joints between neighboring blocks were $5 \mathrm{~mm}$ wide and $75 \mathrm{~mm}$ tall; they were 'filled' up to $2.5 \mathrm{~mm}$ below the surface with a linear elastic material characterized by a Young's modulus of $5 \mathrm{MPa}$ and Poisson's ratio of 0.35 . Such an arrangement represents relatively weak blockto-block interaction. The array of interconnected blocks was supported on a $30 \mathrm{~mm}$ thick layer representing sand bedding, characterized by a Young's modulus of $100 \mathrm{MPa}$ and Poisson's ratio of 0.35. Introduced below the sand bedding was a $400 \mathrm{~mm}$ thick layer representing an aggregate base course, characterized by a Young's modulus of $200 \mathrm{MPa}$ and Poisson's ratio of 0.35 . The base course was supported on a $10 \mathrm{~m}$ thick elastic medium representing subgrade soil that extends to a large depth, characterized by a Young's modulus of $80 \mathrm{MPa}$ and Poisson's ratio of 0.40 . Surface loading was applied in the vertical direction, uniformly spread with intensity $0.5 \mathrm{MPa}$ over a circular region with radius $225 \mathrm{~mm}$. Such loading replicates the contact area of a wide-base truck tire.

Figure 6a provides a general view of the above-described FE model, displaying the individual blocks, the mesh elements, and the loaded region. A three-dimensional Cartesian coordinate system is included, with the $x$ and $y$ axes oriented parallel to the pavement surface and the $\mathrm{z}$-axis pointing downward into the medium. The entire FE system was meshed with 126,806 elements of which 120,006 were three-dimensional 8-node linear brick elements C3D8, and the remaining 6,800 were three-dimensional 8-node linear infinite elements CIN3D8. The latter were attached to the outer edges of the base and subgrade layers, as well as to the bottom of the subgrade layer. All layer and material interfaces were characterized by full bonding. A magnified view of the model near the loaded region is offered in Figure 6b. The FE model dimensions and element sizes were selected based on preliminary convergence study. These selections were validated by comparison against the results of a corresponding LET model after setting the block and joint elastic properties to be the same (and 'filling' the entire joint volume). Two limiting cases were considered in this connection: (i) the elastic properties of the joint material were set to equal the elastic properties of the concrete blocks, and (ii) the elastic properties of the concrete blocks were set to equal the elastic properties of the joint 
material. Further details about the FE model (e.g., element numbers, types, and sizes) are provided in Table 1.

Table 1. Details of the FE model in Figure 6.

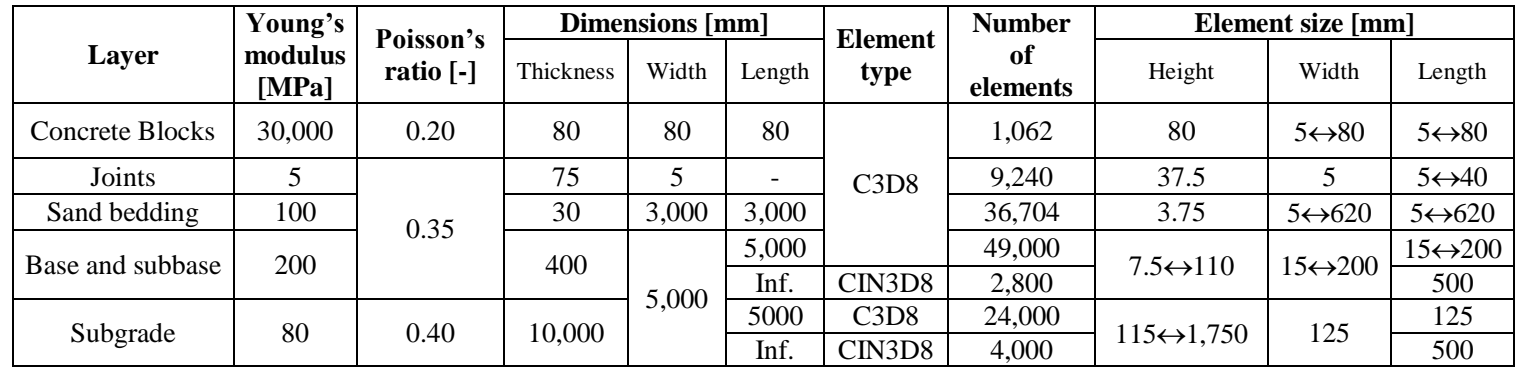

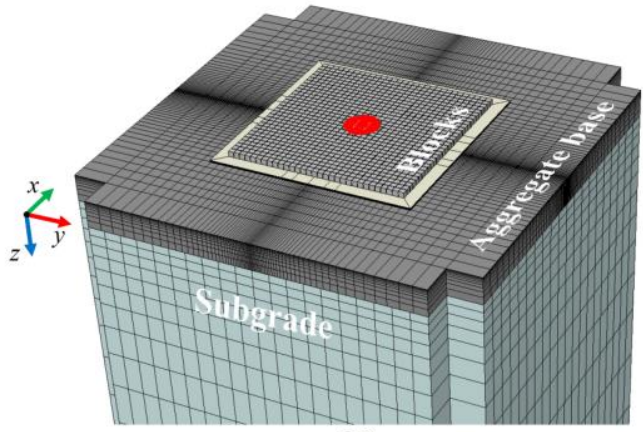

(a)

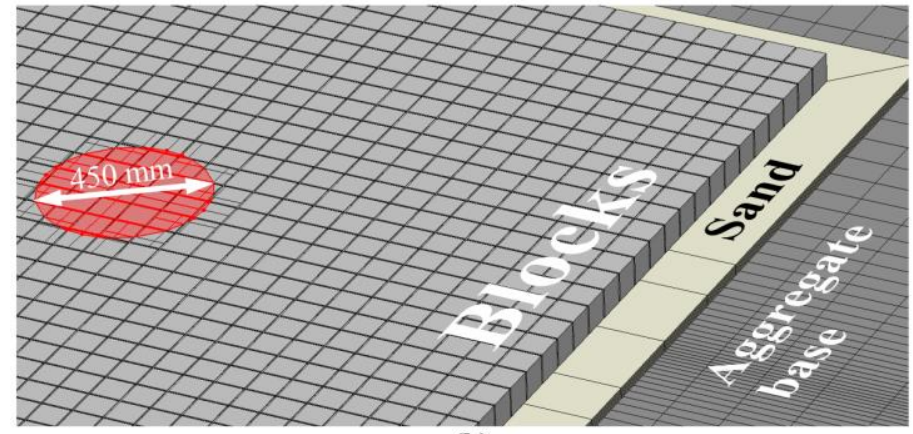

(b)
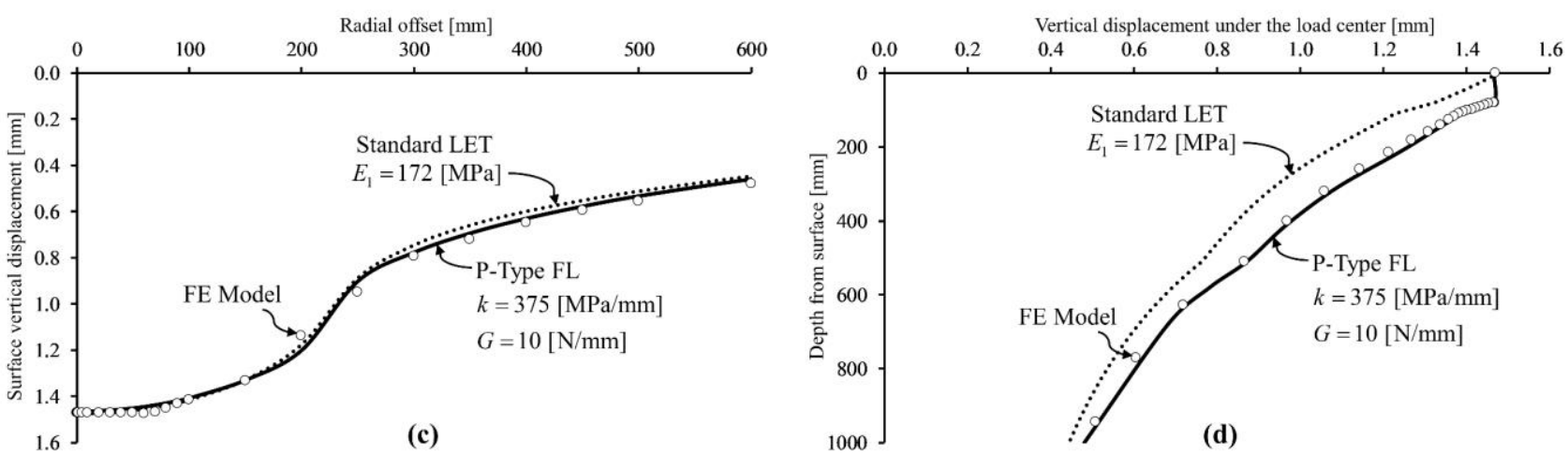

Figure 6. Pavement system surfaced with paving blocks: (a) general view of FE model, (b) magnified view of FE model near the loaded area, (c) radial variation of vertical displacement at the surface, and (d) depth variation of vertical displacement under the load center.

Figure $6 \mathrm{c}$ provides a plot of vertical surface displacements (i.e., deflections) in the FE model for different radial offsets from the load center taken along a $45^{\circ}$ diagonal line in the $x-y$ plane. The FE model results are shown with circular markers. As can be seen, the peak displacement, about 1.45 $\mathrm{mm}$ in magnitude, occurs directly below the load, and diminishes with increased offset distance. This Figure includes two additional curves; the first (dotted line) is a deflection basin created with a 
standard LET model consisting of four layers (top to bottom): $E_{1}=172 \mathrm{MPa}$ representing the $80 \mathrm{~mm}$ layer of blocks and joints, $E_{2}=100 \mathrm{MPa}$ representing the $30 \mathrm{~mm}$ sand layer, $E_{3}=200 \mathrm{MPa}$ representing the $400 \mathrm{~mm}$ aggregate base layer, and $E_{4}=80 \mathrm{MPa}$ representing the subgrade soil. The value of $E_{1}$ is an equivalent modulus, obtained by best matching the FE results. The second curve (solid line) is a deflection basin created in a layered system with properties as listed above except that the top layer, representing the blocks and joints, was a P-type FL. The properties of the FL included: (i) a vertical stiffness $k=375 \mathrm{MPa} / \mathrm{mm}$, simply obtained by dividing the block modulus of $30 \mathrm{GPa}$ by the block height of $80 \mathrm{~mm}$; and (ii) a relatively low characteristic shear interaction $G=10 \mathrm{~N} / \mathrm{mm}$ , obtained by best fitting the FE results. The value of $k_{h}$ is inconsequential given that the FL is at the top.

In Figure 6c, the standard LET matches the FE model with a mean absolute error (MAE) of $33 \mu \mathrm{m}$ while the P-type FL model fits with a MAE of $19 \mu \mathrm{m}$. It graphically apparent that both the standard LET and the layered model with a FL on top can reproduce relatively well the surface displacements calculated from the FE model. Figure 6d provides a plot of vertical displacements in the FE model (circular markers) for different depths up to $1000 \mathrm{~mm}$ below the load center. Two additional curves are superposed over the FE results. The first represents calculated displacements in the standard LET model with an equivalent modulus for the top layer ( $E_{1}=172 \mathrm{MPa}$ ). The second represents displacements in the layered model with a P-type FL on top ( $k=375 \mathrm{MPa} / \mathrm{mm}$ and $G=10 \mathrm{~N} / \mathrm{mm}$ ). As can be seen, the LET model is unable to correctly reproduce the FE results in this case, while the layered model with a FL on top offers a near perfect match to the FE model results (MAE of $10 \mu \mathrm{m}$ ).

The above demonstration of FL superiority over standard LET involved a relatively large tire imprint with a diameter of $450 \mathrm{~mm}$, and relatively small concrete blocks with size (width or length) of $80 \mathrm{~mm}$. For such configuration, the ratio of loading diameter to block size is $5.63(=450 / 80)$. Given that the FL formulation homogenizes the layer of blocks, such ratio can serve as an indication of potential conformity to the underlying FL assumptions in a given situation. Accordingly, higher conformity ratios, e.g., smaller blocks or larger loading zone, would be associated with an improved FL representation, while smaller conformity ratios would be associated with growing disagreement and eventual loss of theory utility. To further investigate this latter point, the entire above-described FE model was executed with increasing block sizes, keeping $80 \mathrm{~mm}$ thick square blocks and $5 \mathrm{~mm}$ joint spacing. The considered block sizes were: $110 \mathrm{~mm}, 140 \mathrm{~mm}, 200 \mathrm{~mm}, 260 \mathrm{~mm}, 320 \mathrm{~mm}$, and 
$440 \mathrm{~mm}$. For a loading diameter of $450 \mathrm{~mm}$ these yield conformity ratios of: 4.09, 3.21, 2.25, 1.73, 1.41, and 1.02 (respectively). Selected results of these added cases are presented in Figure 7.

Figure 7a provides a plot of the vertical surface displacements in the FE model for radial offsets up to $600 \mathrm{~mm}$ taken along a $45^{\circ}$ diagonal line in the $x-y$ plane. Three different deflection curves are shown, each corresponding to a different block size: $80 \mathrm{~mm}$ (same as in Figure 6c), 200 $\mathrm{mm}$, and $320 \mathrm{~mm}$. As can be seen (and expected), a larger block size translates into a smaller peak deflection and is associated with a more tortuous and jagged deflection basin shape. This letter propensity cannot be replicated by a FL model. In this connection, Figure $7 \mathrm{~b}$ shows the attained MAEs as a function of conformity ratio after best matching the different FE results with a P-type FL model. As before, the matching was done by keeping $k=375 \mathrm{MPa} / \mathrm{mm}$ and only manipulating $G$; the values in parentheses are the fitted $G$ 's in units of N/mm. It can be seen that for blocks up to 140 $\mathrm{mm}$ in size the MAEs remain low (about $20 \mu \mathrm{m}$ ) and nominally constant in value. With further increase in block sizes, the MAEs begin to grow exponentially. Based on this chart, it appears that the FL formulation can offer good deflection basin reproducibility for conformity ratios larger than two. A similar analysis (not shown) was done for the ability to match vertical displacements at different depths below the load center (corresponding to Figure 6d). Also in this case it was observed that the FL formulation was able to replicate the FE results for conformity ratios larger than two.
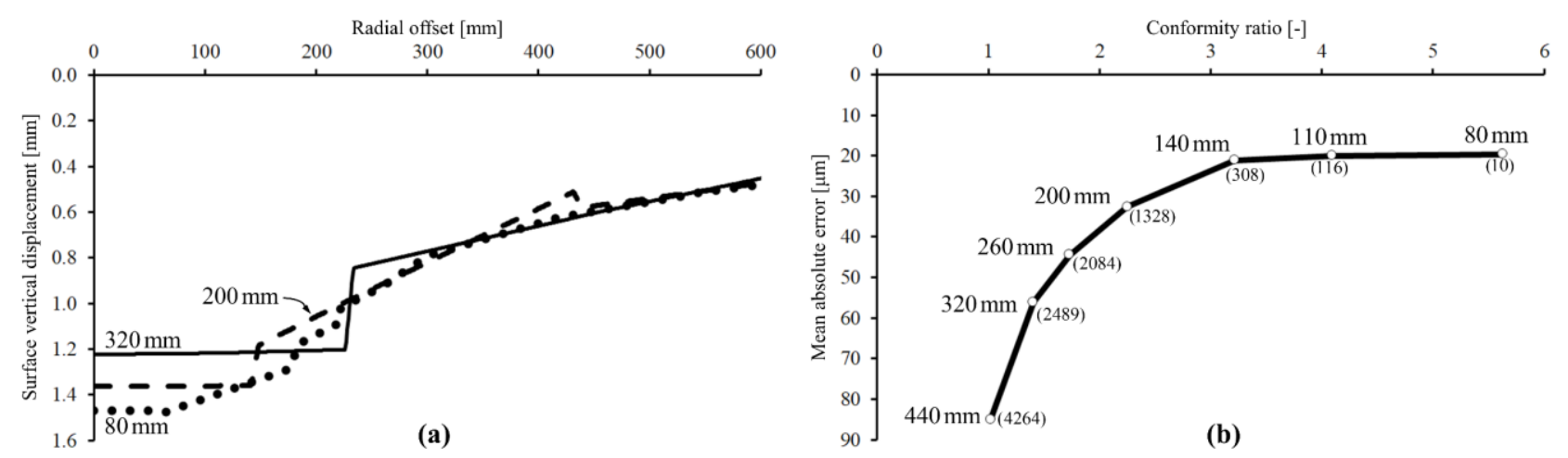

Figure 7. Analysis of FE results for a pavement system surfaced with paving blocks: (a) deflection basins for three different sizes of blocks, and (b) mean absolute error attained after matching the FL formulation (with $k=375 \mathrm{MPa} / \mathrm{mm}$ ) to the FE model results for different conformity ratios (or block sizes). Values in parentheses are the fitted $G$ 's in units of N/mm. 


\section{Summary and discussion}

This paper put forward a new formulation for including a FL within the familiar LET-based analytic pavement model (see Figure 1). The overall aim was to better capture and reproduce, within a rational and familiar engineering framework, the mechanical behavior of a pavement system that includes one or more multi-fractured and discontinuous layers. In this connection, two FL types were suggested: P-type and K-type; each characterized by three independent governing parameters: $k, G$, and $k_{h}$ (see Figure 2). The formulation was based on the assumption that the thickness of the FL can be incorporated as part of the governing parameters, allowing for the FL to be treated as a new kind of interface - see Equation (14). All related equations are fully outlined in Appendix B, so that they can be reproduced and coded by others.

In practice, the governing FL parameters $k$ and $G$ can be derived through inverse analysis, by best-matching results from field tests. The $k$ parameter may also be estimated directly from performing an element test on a representative layer fragment (modulus divided by thickness). It is hard to envision a laboratory technique for obtaining the $G$ parameter. This is because shear deformation resistance offered by a FL depends on the conditions prevailing (in an average sense) at the interfaces between the material blocks. These interfaces are never smooth, may be irregularly shaped, may be randomly contaminated with debris, and are expected to repeatedly open and close as temperatures fluctuate. This latter point also means that even if individual material fragments are insensitive to temperature, a FL is expected to exhibit some thermal sensitivity through the $G$ parameter (see also Levenberg and Rocchi 2020). The $k_{h}$ parameter is as difficult to assess as determining the governing interface bond level in any pavement system. In this connection, a recent state-of-the-art report (Petit et al. 2018) offers a comprehensive review of related modeling approaches as well as measurement methods. Luckily, at least for vertically applied loads, model responses do not appear very sensitive to the choice of $k_{h}$ (see Figures 4 and 5).

The study included a parametric interrogation of two model pavements containing a FL (see Figure 3); it also included comparisons with a FE model (see Figure 6). The aim of both investigations was to demonstrate capabilities and verify the correctness of underlying equations and coding. Results indicate that the new formulation is rich of potential utility. First, when modeling small-element paving technologies, the new theory provides: (i) ability to capture and rationally quantify the interaction level between blocks, and (ii) reproducibility of mechanical responses anywhere within the pavement system. Second, prospective utility is offered by the new formulation for improved 
analytic modeling of: (i) pavement systems that contain cracked-and-seated (or rubblized) PCC, and (ii) aged or rehabilitated pavement systems that contain fatigued (multi-fractured) AC layers.

In all above-mentioned cases, the FL concept is deemed valid on condition that the cracked (or discontinuous) layer contains sufficiently small elements, especially with respect to the applied boundary conditions. A preliminary investigation in this connection (see Figure 7) indicates that the ratio of loading diameter to block size (i.e., conformity ratio) should be at least two for attaining representative results.

\section{REFERENCES}

Ahrlich, R.C. (1989), "Performance and Structural evaluation of Cracked and Seated Concrete," Transportation Research Record, Vol. 1215, pp. 212-218.

Andersen, S., Levenberg, E., and Andersen, M.B. (2018), "Efficient Reevaluation of Surface Displacements in a Layered Elastic Half-space," International Journal of Pavement Engineering, DOI: 10.1080/10298436.2018.1483502.

Baek, C., Thirunavukkarasu, S., Underwood, B.S., Guddati, M.N., and Kim, Y.R. (2012), "Top-down Cracking Prediction Tool for Hot Mix Asphalt Pavements," In A. Scarpas, N. Kringos, and A.L.I. Al-Qadi (eds.), 7th RILEM international conference on Cracking in Pavements. RILEM Bookseries, Vol. 4, pp. 465474, Dordrecht: Springer.

Bemanian, S. and Sebaaly, P. (1999), "Cost-effective Rehabilitation of Portland Cement Concrete Pavement in Nevada," Transportation Research Record, Vol. 1684, pp. 156-164.

Burmister, D.M. (1943), "The Theory of Stresses and Displacements in Layered Systems and Application to the Design of Airport Runways," Proceedings of the Highway Research Board, Vol. 23, Washington, D.C., pp. 126-148.

Burmister, D.M. (1945), "The General Theory of Stresses and Displacements in Layered Systems," Journal of Applied Physics: Vol. 16(2), pp. 89-94 (Part I); Vol. 16(3), pp. 126-127 (Part II); Vol. 16(5), pp. 296-302 (Part III).

De Jong, D.L., Peatz, M.G.F., and Korswagen, A.R. (1973), "Computer Program BISAR, Layered Systems Under Normal and Tangential Loads," External Report, Koninklijke/Shell-Laboratorium, Amsterdam, Netherlands.

Dempsey, J.P., Zhao, Z.G., and Li, H. (1991), “Axisymmetric Indentation of an Elastic Layer Supported by a Winkler Foundation," International Journal of Solids and Structures, Vol. 27(1), pp. 73-87.

Freeman, T.E. (2002), "Evaluation of Concrete Slab Fracturing Techniques in Migrating Reflective Cracking Through Asphalt Overlays," Report VTRC 03-R3, Virginia Transportation Research Council, Charlottesville.

Goodman, J.R. and Popov, E.P. (1968), "Layered Beam Systems with Interlayer Slip," Journal of the Structural Division, Vol. 94(11), pp. 2535-2548. 
Hill, R. (1963), "Elastic Properties of Reinforced Solids: Some Theoretical Principles," Journal of the Mechanics and Physics of Solids, Vol. 11(5), pp. 357-372.

Huang, Y.H. (2004), "Pavement Analysis and Design,” Second Edition, Pearson Prentice Hall, p. 775.

Ioannides A.M. and Khazanovich, L. (1998), "General Formulation for Multilayered Pavement Systems," Journal of Transportation Engineering, Vol. 124(1), pp. 82-90.

Jamshidia, A., Kurumisawab, K., Whitea, G., Nishizawac, T., Igarashib, T., Nawab, T., and Mao, J. (2019), "State-of-the-art of Interlocking Concrete Block Pavement Technology in Japan as a Post-modern Pavement," Construction and Building Materials, Vol. 200, pp. 713-755.

Kachanov, L.M. (1986), "Introduction to Continuum Damage Mechanics,” Martinus Nijhoff Publishers, The Netherlands.

Kai, W. (1987), "Analysis and Calculation of Stresses and Displacements in Layered Elastic Systems," Acta Mechanica Sinica, Vol. 3(3), pp. 251-260.

Kasahara, A. and Matsuno, S. (1988), "Estimation of Apparent Elastic Modulus of Concrete Block Layer," Proceedings of the $3^{\text {rd }}$ International Conference on Concrete Block Paving, Rome, pp. 142-148.

Kerr, A.D. (1964), "Elastic and Viscoelastic Foundation Models,” Journal of Applied Mechanics, Vol. 31(3), pp. 491-498.

Khazanovich, L. and Wang, Q. (2007), "MnLayer: High-performance Layered Elastic Analysis Program,” Transportation Research Record, Vol. 2037, pp. 63-75.

Kutay, M.E. and Lanotte, M. (2018), "Viscoelastic Continuum Damage (VECD) Models for Cracking Problems in Asphalt Mixtures," International Journal of Pavement Engineering, Vol. 19(3), pp. 231-242.

Levenberg, E. (2009), "Backcalculation of Anisotropic Pavement Properties using Time History of Embedded Gauge Readings," Selected Papers From the 2009 GeoHunan International Conference: Asphalt Material Characterization, Accelerated Testing, and Highway Management, ASCE Geotechnical Special Publication (GSP) 190, Walubita, L. F., du Plessis, L., Huang, S-C., Simate, G.S., and Liu, Z. (eds.), American Society of Civil Engineers, Reston, Virginia, pp. 79-85.

Levenberg, E. (2013), "Analysis of Pavement Response to Subsurface Deformations," Computers and Geotechnics, Vol. 50, pp. 79-88.

Levenberg, E. (2016), "Viscoelastic Pavement Modeling with a Spreadsheet," Proceedings of the $8^{\text {th }}$ International Conference on Maintenance and Rehabilitation of Pavements (Mairepav8), Research Publishing, Singapore, pp. 746-755.

Levenberg, E., McDaniel, R.S., Olek, J. (2009), "Validation of NCAT Structural Test Track Experiment using INDOT APT Facility," Publication FHWA/IN/JTRP-2008/26, Joint Transportation Research Program, Indiana Department of Transportation and Purdue University, West Lafayette, IN.

Levenberg, E., and Rocchi, I. (2020), "On the Thermal Sensitivity of Unbound Granular Pavement Layers," International Journal of Pavement Research and Technology, Vol. 13(1), pp. 32-39.

Longman, I.M., (1956), "Note on a Method for Computing Infinite Integrals of Oscillatory Functions," Mathematical Proceedings of the Cambridge Philosophical Society, Vol. 52(4), pp. 764-768. 
Love, A.E.H. (1927), "Treatise on the Mathematical Theory of Elasticity", $4^{\text {th }}$ Edition, Cambridge University Press, UK.

Maina, J.W. and Matsui, K. (2004), "Developing Software for Elastic Analysis of Pavement Structure Responses to Vertical and Horizontal Surface Loadings," Transportation Research Record, Vol. 1896, pp. 107118.

Molenaar, A.A.A., Moll, H.O., and Houben L.J.M. (1984), "Structural Model for Concrete Block Pavement," Transportation Research Record, Vol. 954, pp. 16-27.

Nishizawa, T. (2003), "A Tool for Structural Analysis of Block Pavements based on 3DFEM," Proceedings of the $7^{\text {th }}$ International Conference on Concrete Block Paving, Sun City, South Africa, pp. 226-232; Concrete Massonary Association (CMA).

Pan, E. (1989), "Static Response of a Transversely Isotropic and Layered Half-space to General Surface Loads," Physics of the Earth and Planetary Interiors Vol. 54(3), pp. 353-363.

Petit, C., Chabot, A., Destree, A., and Raab, C. (2018), “Interface Debonding Behaviour.” In W.G. Buttlar, A. Chabot, E. V. Dave, C. Petit, \& G. Tebaldi (Eds), Mechanisms of Cracking and Debonding in Asphalt and Composite Pavements. Chapter III of the state-of-the-art report of the RILEM Technical Committee 241-MCD Series, Vol. 28, pp. 103-154. Cham: Springer, ISBN 978-3-319-76848-9, Series ISSN, 2213-204X doi:10.1007/978-3-319-76849-6_3.

Peutz, M.G.F, Van Kempen, H.P.M., and Jones, A. (1968), "Layered Systems under Normal Surface Loads," Highway Research Record, Vol. 228, pp. 34-45.

Schapery, R.A. (1999), "Nonlinear Viscoelastic and Viscoplastic Constitutive Equations with Growing Damage," International Journal of Fracture, Vol. 97, pp. 33-66.

Schneider, W.C. (1969), "Contact Problems in Elasticity and the Solution for a Thick Layer on a Winkler Type Foundation," NASA Technical Memorandum TM X-58023, National Aeronautics And Space Administration, Manned Spacecraft Center, Houston, TX.

Singh, S.J. (1986), "Static Deformation of a Transversely Isotropic Multilayered Halfspace by Surface Loads," Physics of the Earth and Planetary Interiors, Vol. 42(4), pp. 263-273.

Skar, A. and Poulsen, P.N. (2015), "3-D Cohesive Finite Element Model for Application in Structural Analysis of Heavy Duty Composite Pavements," Construction and Building Materials, Vol. 101(Part 1), pp. 417-431.

Skar, A., Ingeman-Nielsen, T., and Levenberg, E. (2018), "Mechanistic Analysis of Frost Action under Pavements," Proceedings of the AIC2018 Conference: Transportation Infrastructure Engineering in Cold Regions, pp. 76-77.

Van Cauwelaert, F. (2003), "Pavement Design and Evaluation-The Required Mathematics and its Applications," M. Stet (ed). Federation of the Belgian Cement Industry, Brussels, Belgium.

Witczak, M.W. and Rada, G.R. (1992), "Nationwide Evaluation Study of Asphalt Concrete Overlays Placed on Fractured Portland Cement Concrete Pavements," Transportation Research Record, Vol. 1374, pp. 19-26. 


\section{APPENDIX A}

This Appendix outlines the expressions obtained after inserting $\varphi_{i}$ from Equation (12) into Equations (6)-(11). These allow for the calculation of stresses and displacements anywhere within a LET model.

$$
\begin{aligned}
& \left(\sigma_{z}\right)_{i}=q_{0} \bar{a} \int_{m=0}^{\infty} J_{0}(m \bar{r})\left(\begin{array}{l}
\left(-A_{i}+C_{i}\left(1-2 v_{i}-m \bar{z}\right)\right) e^{-m\left(\bar{z}_{i}-\bar{z}\right)} \\
-\left(B_{i}+D_{i}\left(1-2 v_{i}+m \bar{z}\right)\right) e^{-m\left(\bar{z}-\bar{z}_{i-1}\right)}
\end{array}\right) J_{1}(m \bar{a}) d m
\end{aligned}
$$

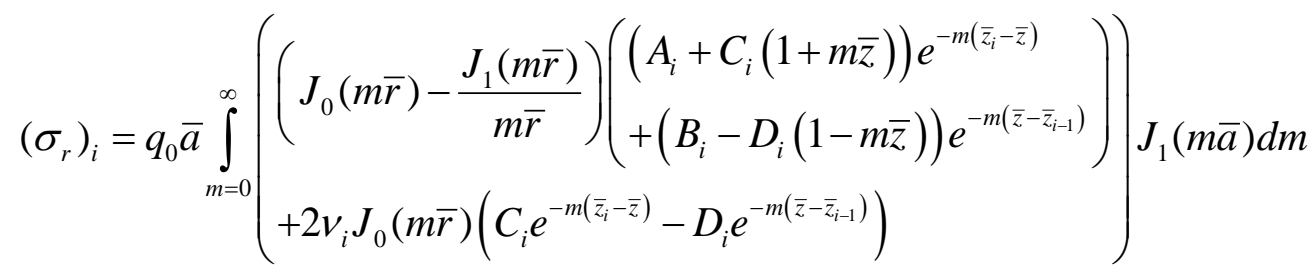

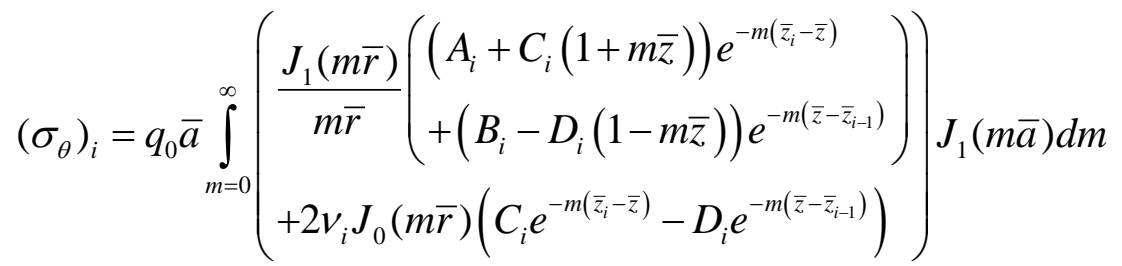

$$
\begin{aligned}
& \left(\tau_{r z}\right)_{i}=q_{0} \bar{a} \int_{m=0}^{\infty} J_{1}(m \bar{r})\left(\begin{array}{l}
\left(A_{i}+C_{i}\left(2 v_{i}+m \bar{z}\right)\right) e^{-m\left(\bar{z}_{i}-\bar{z}\right)} \\
-\left(B_{i}-D_{i}\left(2 v_{i}-m \bar{z}\right)\right) e^{-m\left(\bar{z}-\bar{z}_{i-1}\right)}
\end{array}\right) J_{1}(m \bar{a}) d m \\
& \left(u_{z}\right)_{i}=\frac{q_{0} \bar{a} H\left(1+v_{i}\right)}{E_{i}} \int_{m=0}^{\infty} \frac{J_{0}(m \bar{r})}{m}\left(\begin{array}{l}
\left(A_{i}-C_{i}\left(2-4 v_{i}-m \bar{z}\right)\right) e^{-m\left(\bar{z}_{i}-\bar{z}\right)} \\
-\left(B_{i}+D_{i}\left(2-4 v_{i}+m \bar{z}\right)\right) e^{-m\left(\bar{z}-\bar{z}_{i-1}\right)}
\end{array}\right) J_{1}(m \bar{a}) d m \\
& \left(u_{r}\right)_{i}=-\frac{q_{0} \bar{a} H\left(1+v_{i}\right)}{E_{i}} \int_{m=0}^{\infty} \frac{J_{1}(m \bar{r})}{m}\left(\begin{array}{c}
\left(A_{i}+C_{i}(1+m \bar{z})\right) e^{-m\left(\bar{z}_{i}-\bar{z}\right)} \\
+\left(B_{i}-D_{i}(1-m \bar{z})\right) e^{-m\left(\bar{z}-\bar{z}_{i-1}\right)}
\end{array}\right) J_{1}(m \bar{a}) d m
\end{aligned}
$$




\section{APPENDIX B}

This Appendix presents the matrices $\mathbf{M}$ and $\mathbf{N}$ in Equation (14) for the standard LET formulation as well as for the two fragmented layer formulations: (i) P-type, denoted as $\mathbf{M}^{\mathrm{P}}$ and $\mathbf{N}^{\mathrm{P}}$, and (ii) $\mathrm{K}$ type, denoted as $\mathbf{M}^{\mathrm{K}}$ and $\mathbf{N}^{\mathrm{K}}$. As a means to constrain the expressions to a manageable and presentable size, the following symbols are introduced

$$
\begin{aligned}
& F_{i}=e^{-m\left(\bar{z}_{i}-\bar{z}_{i-1}\right)} \\
& S_{i}=\left(1+v_{i}\right) / E_{i} \\
& \bar{G}=G / H
\end{aligned}
$$

\section{Standard LET formulation}

$$
\begin{aligned}
\mathbf{M} & =\left[\begin{array}{cccc}
1 & F_{i} & m \bar{z}_{i}+2 v_{i}-1 & \left(m \bar{z}_{i}-2 v_{i}+1\right) F_{i} \\
1 & -F_{i} & m \bar{z}_{i}+4 v_{i}-2 & \left(4 v_{i}-2-m \bar{z}_{i}\right) F_{i} \\
1 & -F_{i} & 2 v_{i}+m \bar{z}_{i} & \left(2 v_{i}-m \bar{z}_{i}\right) F_{i} \\
1 & F_{i} & 1+m \bar{z}_{i} & \left(m \bar{z}_{i}-1\right) F_{i}
\end{array}\right] \\
\mathbf{N} & =\left[\begin{array}{cccc}
-F_{i+1} & -1 & \left(1-2 v_{i+1}-m \bar{z}_{i}\right) F_{i+1} & 2 v_{i+1}-1-m \bar{z}_{i} \\
-\frac{S_{i+1} F_{i+1}}{S_{i}} & \frac{S_{i+1}}{S_{i}} & \left(2-4 v_{i+1}-m \bar{z}_{i}\right) \frac{S_{i+1} F_{i+1}}{S_{i}} & \left(2-4 v_{i+1}+m \bar{z}_{i}\right) \frac{S_{i+1}}{S_{i}} \\
-F_{i+1} & 1 & -\left(2 v_{i+1}+m \bar{z}_{i}\right) F_{i+1} & m \bar{z}_{i}-2 v_{i+1} \\
-\frac{S_{i+1} F_{i+1}}{S_{i}} & -\frac{S_{i+1}}{S_{i}} & -\left(1+m \bar{z}_{i}\right) \frac{S_{i+1} F_{i+1}}{S_{i}} & \left(1-m \bar{z}_{i}\right) \frac{S_{i+1}}{S_{i}}
\end{array}\right]
\end{aligned}
$$

\section{P-type fragmented layer}

$\mathbf{M}^{\mathrm{P}}=\left[\begin{array}{cccc}M_{11}^{\mathrm{P}} & M_{12}^{\mathrm{P}} & M_{13}^{\mathrm{P}} & M_{14}^{\mathrm{P}} \\ -\frac{k H}{m} S_{i} & \frac{k H}{m} S_{i} F_{i} & \frac{k H}{m}\left(2-4 v_{i}-m \bar{z}_{i}\right) S_{i} & \frac{k H}{m}\left(2-4 v_{i}+m \bar{z}_{i}\right) S_{i} F_{i} \\ 1 & -F_{i} & 2 v_{i}+m \bar{z}_{i} & \left(2 v_{i}-m \bar{z}_{i}\right) F_{i} \\ -\frac{k_{h} H}{m} S_{i}-1 & M_{42}^{\mathrm{P}} & M_{43}^{\mathrm{P}} & M_{44}^{\mathrm{P}}\end{array}\right]$

$$
M_{11}^{\mathrm{P}}=-1-\left(k H m^{-1}+0.5 m \bar{G}\right) S_{i}
$$




$$
\begin{aligned}
& M_{12}^{\mathrm{P}}=\left(\left(k H m^{-1}+0.5 m \bar{G}\right) S_{i}-1\right) F_{i} \\
& M_{13}^{\mathrm{P}}=1-2 v_{i}-m \bar{z}_{i}+\left(\left(1-2 v_{i}\right)\left(2 k H m^{-1}+\bar{G} m\right)-\bar{z}_{i}\left(k H+0.5 m^{2} \bar{G}\right)\right) S_{i} \\
& M_{14}^{\mathrm{P}}=\left(2 v_{i}-1-m \bar{z}_{i}+\left(k H m^{-1}\left(m \bar{z}_{i}-4 v_{i}+2\right)+0.5 m \bar{G}\left(m \bar{z}_{i}-4 v_{i}+2\right)\right) S_{i}\right) F_{i} \\
& M_{42}^{\mathrm{P}}=\left(1-k_{h} H m^{-1} S_{i}\right) F_{i} \\
& M_{43}^{\mathrm{P}}=-k_{h} H m^{-1} S_{i}\left(1+m \bar{z}_{i}\right)-2 v_{i}-m \bar{z}_{i} \\
& M_{44}^{\mathrm{P}}=\left(k_{h} H m^{-1} S_{i}\left(1-m \bar{z}_{i}\right)-2 v_{i}+m \bar{z}_{i}\right) F_{i} \\
& \mathbf{N}^{\mathrm{P}}=\left[\begin{array}{cccc}
\frac{k H}{m} S_{i+1} F_{i+1} & -\frac{k H}{m} S_{i+1} & N_{13}^{\mathrm{P}} & N_{14}^{\mathrm{P}} \\
N_{21}^{\mathrm{P}} & N_{22}^{\mathrm{P}} & N_{23}^{\mathrm{P}} & N_{24}^{\mathrm{P}} \\
-F_{i+1} & 1 & -\left(2 v_{i+1}+m \bar{z}_{i}\right) F_{i+1} & m \bar{z}_{i}-2 v_{i+1} \\
\frac{k_{h} H}{m} S_{i+1} F_{i+1} & \frac{k_{h} H}{m} S_{i+1} & \frac{k_{h} H}{m}\left(m \bar{z}_{i}+1\right) S_{i+1} F_{i+1} & \frac{k_{h} H}{m}\left(m \bar{z}_{i}-1\right) S_{i+1}
\end{array}\right] \\
& N_{13}^{\mathrm{P}}=k H m^{-1}\left(4 v_{i+1}-2+m \bar{z}_{i}\right) S_{i+1} F_{i+1} \\
& N_{14}^{\mathrm{P}}=k H m^{-1}\left(4 v_{i+1}-2-m \bar{z}_{i}\right) S_{i+1} \\
& N_{21}^{\mathrm{P}}=\left(\left(k H m^{-1}+0.5 m \bar{G}\right) S_{i+1}-1\right) F_{i+1} \\
& N_{22}^{\mathrm{P}}=-\left(k H m^{-1}+0.5 m \bar{G}\right) S_{i+1}-1 \\
& N_{23}^{\mathrm{P}}=\left(k H m^{-1}\left(4 v_{i+1}+m \bar{z}_{i}-2\right) S_{i+1}+0.5 m \bar{G}\left(4 v_{i+1}+\bar{z}_{i} m-2\right) S_{i+1}-2 v_{i+1}-m \bar{z}_{i}+1\right) F_{i+1} \\
& N_{24}^{\mathrm{P}}=k H m^{-1}\left(4 v_{i+1}-m \bar{z}_{i}-2\right) S_{i+1}+0.5 m \bar{G}\left(4 v_{i+1}-m \bar{z}_{i}-2\right) S_{i+1}+2 v_{i+1}-m \bar{z}_{i}-1
\end{aligned}
$$




\section{K-type fragmented layer}

$$
\begin{aligned}
& \mathbf{M}^{\mathrm{K}}=\left[\begin{array}{cccc}
M_{11}^{\mathrm{K}} & M_{12}^{\mathrm{K}} & M_{13}^{\mathrm{K}} & M_{14}^{\mathrm{K}} \\
-\frac{k H}{m} S_{i} & \frac{k H}{m} S_{i} F_{i} & \frac{k H}{m}\left(2-4 v_{i}-m \bar{z}_{i}\right) S_{i} & \frac{k H}{m}\left(2-4 v_{i}+m \bar{z}_{i}\right) S_{i} F_{i} \\
1 & -F_{i} & 2 v_{i}+m \bar{z}_{i} & \left(2 v_{i}-m \bar{z}_{i}\right) F_{i} \\
-\frac{k_{h} H}{m} S_{i}-1 & M_{42}^{\mathrm{P}} & M_{43}^{\mathrm{P}} & M_{44}^{\mathrm{P}}
\end{array}\right] \\
& M_{11}^{\mathrm{K}}=-k H m^{-1} S_{i}-\frac{m \bar{G}}{2}\left(S_{i}+\frac{m}{2 H k}\right)-1 \\
& M_{12}^{\mathrm{K}}=\left(k H m^{-1} S_{i}+\frac{m \bar{G}}{2}\left(S_{i}-\frac{m}{2 H k}\right)-1\right) F_{i} \\
& M_{13}^{\mathrm{K}}=\left(1-2 v_{i}\right)\left(\left(m \bar{G}+\frac{2 k H}{m}\right) S_{i}+1\right)+\frac{m^{2} \bar{G}}{2}\left(\frac{1-2 v_{i}}{2 k H}-\bar{z}_{i} S_{i}\right)-m \bar{z}_{i}\left(\frac{m^{2} \bar{G}}{4 k H}+\frac{k H}{m} S_{i}+1\right) \\
& M_{14}^{\mathrm{K}}=\left(\left(2 v_{i}-1\right)\left(\frac{\bar{G} m^{2}}{4 k H}-\left(\bar{G} m+\frac{2 k H}{m}\right) S_{i}+1\right)+\left(k H \bar{z}_{i}+\frac{m^{2} \bar{G} \overline{z_{i}}}{2}\right) S_{i}-\frac{m^{3} \bar{G} \bar{z}_{i}}{4 k H}-m \bar{z}_{i}\right) F_{i} \\
& \mathbf{N}^{\mathrm{K}}=\left[\begin{array}{cccc}
\frac{k H}{m} S_{i+1} F_{i+1} & -\frac{k H}{m} S_{i+1} & N_{13}^{\mathrm{P}} & N_{14}^{\mathrm{P}} \\
N_{21}^{\mathrm{K}} & N_{22}^{\mathrm{K}} & N_{23}^{\mathrm{K}} & N_{24}^{K} \\
-F_{i+1} & 1 & -\left(2 v_{i+1}+m \bar{z}_{i}\right) F_{i+1} & m \bar{z}_{i}-2 v_{i+1} \\
\frac{k_{h} H}{m} S_{i+1} F_{i+1} & \frac{k_{h} H}{m} S_{i+1} & \frac{k_{h} H}{m}\left(m \bar{z}_{i}+1\right) S_{i+1} F_{i+1} & \frac{k_{h} H}{m}\left(m \bar{z}_{i}-1\right) S_{i+1}
\end{array}\right] \\
& N_{21}^{\mathrm{K}}=\left(\left(k H m^{-1}+0.5 m \bar{G}\right) S_{i+1}-\frac{m^{2} \bar{G}}{4 k H}-1\right) F_{i+1} \\
& N_{22}^{\mathrm{K}}=-\left(k H m^{-1}+0.5 m \bar{G}\right) S_{i+1}-\frac{m^{2} \bar{G}}{4 k H}-1 \\
& N_{23}^{\mathrm{K}}=\left(\begin{array}{l}
\left(2 v_{i+1}-1\right)\left(\left(2 k H m^{-1}+m \bar{G}\right) S_{i+1}-1\right) \\
+\frac{m^{2} \bar{G}}{2}\left(\bar{z}_{i} S_{i+1}-\frac{\left(2 v_{i+1}-1\right)}{2 k H}-\frac{m \bar{z}_{i}}{2 k H}\right)+k H \bar{z}_{i} S_{i+1}-m \bar{z}_{i}
\end{array}\right) F_{i+1}
\end{aligned}
$$




$$
N_{24}^{\mathrm{K}}=-\left(\begin{array}{l}
\frac{m^{2} \bar{G}}{4}\left(\left(\frac{m}{k H}+2 S_{i+1}\right) \bar{z}_{i}+\frac{1-2 v_{i+1}}{k H}\right) \\
+k H \bar{z}_{i} S_{i+1}+m \bar{z}_{i}+\left(1-2 v_{i+1}\right)\left(\left(2 k H m^{-1}+m \bar{G}\right) S_{i+1}+1\right)
\end{array}\right)
$$

